\title{
Análise in silico do polimorfismo rs1803909 do gene $A N X A 2$ expresso em monócitos de sangue periférico e sua relação com a osteoporose humana
}

\author{
In silico analysis of the rs 1803909 polymorphysis of the ANXA2 gene expressed in peripheral blood \\ monocytes and its association with human osteoporosis
}

Análisis in silico del polimorfismo rs1803909 del gen ANXA2 expresado en monocitos de sangre periférica y su relación con la osteoporosis humana

Recebido: 04/12/2021 | Revisado: 11/12/2021 | Aceito: 18/12/2021 | Publicado: 02/01/2022

\author{
Rubens Barbosa Rezende \\ ORCID: https://orcid.org/0000-0002-5421-0519 \\ Faculdade UniBF, Brasil \\ E-mail: rubensrezende420@gmail.com \\ Larissa Teodoro Rabi \\ ORCID: https://orcid.org/0000-0001-5584-8429 \\ Universidade Estadual de Campinas, Brasil \\ E-mail: larissateodororabi@gmail.com
}

\begin{abstract}
Resumo
Objetivou-se avaliar as possíveis alterações morfofuncionais e de estabilidade proteica decorrentes das alterações dos aminoácidos Tirosina por uma Histidina na posição 269, bem como, correlacionar com a função fisiológica da proteína e sua provável ligação com a osteoporose humana. Por meio de uma análise in silico com base nas informações disponíveis nos bancos de dados NCBI dbSNP (mudança de aminoácidos e posição) e UNIPROT (sequência na proteína). Os impactos da modificação Y269H foram analisados a partir das ferramentas SIFT, AlignGVGD, SNAP e PROVEAN (função e estrutura), e PolyPhen-2 (natureza da alteração). Além disso, utilizou-se também a ferramenta MuPRO (alterações de estabilidade na proteína). A análise in silico do polimorfismo rs1803909 demonstrou alteração funcional (ferramenta SIFT, Score= 0). Bem como, estima-se que a troca de aminoácidos pode estar relacionada a alterações danosas (PolyPhen-2, Score= 0,993) e associada a modificações na função da proteína (PROVEAN, Score= -4.015). Além disso, foram observados impactos estruturais (Align-GVGD, Score= 83,33, Classe C65) e funcionais (SNAP, Score= 57). De forma complementar, observou-se diminuição da estabilidade proteica decorrente da alteração Y269H pela ferramenta MuPRO, $\Delta \Delta \mathrm{G}=-1.6731749$. Contudo, as alterações morfofuncionais podem estar ligadas a processos danosos e a diminuição de estabilidade da proteína, dificultando assim a sua ação. Além disso, a compreensão das alterações morfofuncionais e de estabilidade do rs 1803909 podem auxiliar na busca por marcadores genéticos e moleculares de diagnóstico precoce para a osteoporose em humanos.
\end{abstract}

Palavras-chave: Cálcio; Circulação sanguínea; Osteoporose; Polimorfismo de nucleotídeo único; Sangue.

\begin{abstract}
The objective was to evaluate the possible morphofunctional and protein stability alterations resulting from Tyrosine amino acid changes by a Histidine at position 269 , as well as to correlate with the physiological function of the protein and its probable association with human osteoporosis. Through an in silico analysis based on the information available in the NCBI dbSNP (amino acid change and position) and UNIPROT (sequence in the protein) databases. The impacts of the $\mathrm{Y} 269 \mathrm{H}$ modification were analyzed from the tools SIFT, Align-GVGD, SNAP and PROVEAN (function and structure), and PolyPhen-2 (nature of the change). In addition, MuPRO tool (stability changes in the protein) were also used. In silico analysis of the $r s 1803909$ polymorphism demonstrated functional alteration (SIFT tool, Score $=0$ ). As well as, it is estimated that amino acid exchange may be related to damaging alterations (PolyPhen-2, Score $=0.993)$ and associated with modifications in protein function $($ PROVEAN, Score $=-4.015)$. In addition, structural (Align-GVGD, Score $=83.33$, Class C65) and functional (SNAP, Score $=57$ ) impacts were observed. Complementarily, decreased protein stability arising from $\mathrm{Y} 269 \mathrm{H}$ alteration was observed by MuPRO tool, $\Delta \Delta \mathrm{G}=-1.6731749$. However, morphofunctional alterations may be connected to damaging processes and the decreased stability of the protein, thus hindering its action. Furthermore, understanding the morphofunctional and stability changes of rs 1803909 may aid in the search for early diagnostic genetic and molecular markers for osteoporosis in humans.
\end{abstract}

Keywords: Blood circulation; Blood; Calcium; Osteoporosis; Polymorphism, single nucleotide. 


\begin{abstract}
Resumen
El objetivo es evaluar las posibles alteraciones morfofuncionales y de estabilidad proteica que conllevan las modificaciones de los aminoácidos de la Tirosina por una Histidina en la posición 269, así como correlacionarlas con la función fisiológica de la proteína y su probable relación con la osteoporosis humana. Mediante un análisis in silico basado en la información disponible en las bases de datos NCBI dbSNP (cambio y posición de aminoácidos) y UNIPROT (secuencia en la proteína). Los impactos de la modificación Y269H se analizaron a partir de las herramientas SIFT, Align-GVGD, SNAP y PROVEAN (función y estructura), y PolyPhen-2 (naturaleza del cambio). Además, se utilizaron la herramienta MuPRO (cambios de estabilidad en la proteína). El análisis in silico del polimorfismo rs 1803909 demostró una alteración funcional (herramienta SIFT, Puntuación= 0). Además, se estima que el intercambio de aminoácidos puede estar relacionado con cambios perjudiciales (PolyPhen-2, Puntuación= 0,993) y asociado a modificaciones en la función de las proteínas (PROVEAN, Puntuación= -4,015). Además, se observaron impactos estructurales (Align-GVGD, Puntuación= 83,33, Clase C65) y funcionales (SNAP, Puntuación= 57). Complementariamente, se observó una disminución de la estabilidad de la proteína derivada de la alteración Y269H mediante la herramienta MuPRO, $\Delta \Delta \mathrm{G}=-1,6731749$. Sin embargo, las alteraciones morfofuncionales pueden estar vinculadas a procesos perjudiciales y a la disminución de la estabilidad de la proteína, dificultando así su acción. Además, la comprensión de las alteraciones morfofuncionales y de estabilidad del rs1803909 puede ayudar en la búsqueda de marcadores genéticos y moleculares de diagnóstico precoz de la osteoporosis en humanos.
\end{abstract}

Palabras clave: Calcio; Circulación sanguínea; Osteoporosis; Polimorfismo de nucleótido simple; Sangre.

\title{
1. Introdução
}

A osteoporose é considerada a doença óssea mais comum no mundo, e é caracterizada por baixa massa óssea, consequente degradação da microestrutura de seu tecido e diminuição de sua resistência. A massa óssea adulta é derivada do pico atingido durante a adolescência e mantida até que o ciclo de remodelação óssea - geralmente um processo fortemente acoplado - que ocorre e altere o equilíbrio entre os osteoblastos e os osteoclastos de reabsorção (Riggs \& Melton, 1983; Lane $\&$ Nydick, 1999). As estimativas indicaram que 50\% do sexo feminino e $20 \%$ do masculino acima dos 50 anos, sofreram fraturas relacionadas à osteoporose (Coughlan \& Dockery, 2014).

O gene $A N X A 2$, é constituído por 17 éxons distribuídos ao longo de 40Kb de DNA genômico no cromossomo 15 (15q22.2), e que codifica a proteína Anexina A2 (ANX2), possui 339 aminoácidos e 38,6 kDa (Hedhli, et al., 2012), está localizado em uma variedade de tipos de células, incluindo as endoteliais, trofoblásticas, epiteliais e tumorais, bem como células imunes inatas, como macrófagos, monócitos e dendríticas. O ANXA2 é capaz de existir na forma monomérica ou heterotetramérica (Cesarman, Guevara, Hajjar, 1994; Hajjar, Jacovina, Chacko, 1994). E o polimorfismo rs1803909 está localizado na região chr15:60351225 (GRCh38.p13) do gene ANXA2, e corresponde a uma troca A>G promovendo a alteração de aminoácidos de uma Tirosina por uma Histidina na posição 269.

Este trabalho se justifica na importância de se compreender o impacto do polimorfismo rs1803909 no gene ANXA2 e sua relação com a possível gênese da osteoporose humana, uma vez que já se tem listado na literatura a associação de polimorfismos deste gene à osteoporose. Dessa forma, objetivou-se avaliar as possíveis alterações morfofuncionais e de estabilidade proteica decorrentes das alterações dos aminoácidos Tirosina por uma Histidina na posição 269, bem como, correlacionar com a função fisiológica da proteína e sua provável ligação com a osteoporose humana.

\section{Metodologia}

Realizou-se uma análise in silico, com base nas informações disponíveis nos bancos de dados NCBI dbSNP (https://www.ncbi.nlm.nih.gov/snp/) no qual avalia a mudança de aminoácidos e suas posições e, UNIPROT (https://www.uniprot.org/) verificando a sequência de proteínas e informações funcionais. Bem como, os impactos da modificação Y269H foram analisados a partir das ferramentas: SIFT ( $\mathrm{Ng}$ \& Henikoff, 2003) que estima o efeito da substituição de aminoácidos no papel proteico com base na homologia e nas propriedades químicas dos aminoácidos. A AlignGVGD (Mathe, et al., 2006; Tavtigian, et al., 2006) (http://agvgd.hci.utah.edu/) combina as propriedades biofísicas de 
aminoácidos e o alinhamento de muitas sequências proteicas para prever que as substituições missense nos genes de interesse. A SNAP (Bromberg \& Rost, 2007) avalia as mudanças na estrutura secundária das proteínas e compara a acessibilidade dos solventes quando os aminoácidos são alterados por meio de métodos de Neural Network. Já a ferramenta PROVEAN (Choi \& Chan, 2015) (http://provean.jcvi.org/index.php) é usada para verificação de função da proteína com base na pontuação de alinhamento de sequências; e o PolyPhen-2 (Adzhubei, et al., 2010) avalia o impacto na estrutura e função das proteínas por meio de métodos de análise empírica e comparando as características físicas das moléculas. Além disso, utilizou-se também a ferramenta MuPRO (Cheng, et al., 2006) (http://mupro.proteomics.ics.uci.edu/) na qual prevê como a mutação nos aminoácidos de um único local, acomete a estabilidade proteica.

Bem como, a correlação entre as alterações de estabilidade e morfofuncionais identificadas na análise in silico com o comportamento fisiopatológico da proteína foram executadas a partir da busca de artigos científicos na base de dados PUBMED, através dos descritores: "Osteoporosis", "Polymorphism, Single Nucleotide" e "Blood Circulation", cadastrados no DeCS/MeSH, e utilizando os operadores booleanos AND e OR. Como também, foi feita uma busca manual, utilizando os descritores: "Peripheral blood monocyte" e "ANXA2 gene".

\section{Resultados e Discussão}

A análise in silico do polimorfismo rs 1803909 demonstrou alteração funcional (ferramenta SIFT, Score= 0). Bem como, estima-se que a troca de aminoácidos pode estar relacionada a alterações danosas (PolyPhen-2, Score $=0,993$ ) e relacionada a alterações na função da proteína (PROVEAN, Score= -4.015). Além disso, foram observados impactos estruturais (Align-GVGD, Score= 83,33, Classe C65) e funcionais (SNAP, Score $=57$ ).

O grau de impacto funcional pode variar, portanto, a classificação da classe não indica o tamanho do impacto funcional do polimorfismo, pelo contrário, apenas indica a extensão em que o impacto é investigado. Além disso, a categoria de polimorfismo não significa sua importância em uma determinada população; o polimorfismo pode ter um grande impacto em sua função corporal (Blakely, 2005; Zhang, et al., 2005).

De forma complementar, observou-se diminuição da estabilidade proteica decorrente da alteração Y269H pela ferramenta MuPRO, $\Delta \Delta \mathrm{G}=-1.6731749$. Contudo, as proteínas são as principais responsáveis pelas funções dos genes em organismos biológicos. E alterações em suas condições fisiológicas geralmente se refletem em mudanças na expressão e/ou metabolismo (Kesisis, et al., 2010; De Jong, et al., 2010; Kuo, et al., 2011).

Para começar a vincular associações de polimorfismos de nucleotídeos únicos (SNP) com efeitos funcionais potenciais, é proposto um sistema de classificação que designa o grau em que um determinado SNP demonstrou ter um papel funcional, mais notavelmente nos comportamentos que causam doenças. No entanto, a classificação proposta não determina o grau de impacto funcional do polimorfismo, portanto, é impossível prever a probabilidade de um SNP específico associado à uma doença, por exemplo (Chorley, et al., 2008, Sadee, et al., 2011; Rezende \& Teodoro, 2021).

Para entender a base mecanicista dos polimorfismos relacionados a fenótipos específicos ou resultados comportamentais, é necessário entender se eles são funcionais (isto é, se altera a função de um gene ou de um grupo de genes). $\mathrm{Na}$ maioria dos casos, a função do polimorfismo relevante não é definida e deve ser assumida ou inferida como o efeito no gene que contém o polimorfismo. Em casos raros, o polimorfismo pode ser uma variação não sinônima na região codificadora, que altera a estrutura do gene da proteína do produto (Chorley, et al., 2008; Rezende \& Teodoro, 2021).

Os polimorfismos mais comuns são os reguladores potenciais, localizados em regiões não codificantes, incluindo regiões promotoras/de montante, de jusante e íntron, que podem afetar a transcrição (Chorley, et al., 2008); no íntron e regiões não traduzidas transcritas como o RNA, podem afetar a transcrição, emenda de RNA, estabilidade ou tradução (Sadee, et al., 
2011); ou em regiões intergênicas com funções desconhecidas (Liao \& Lee, 2010). Um único SNP é capaz de ter um impacto funcional mínimo, mas pode estar relacionado a um desequilíbrio em um conjunto de polimorfismos que formam haplótipos ligado ao resultado funcional da expressão ou função do gene (Drysdale, et al., 2000; Rezende \& Teodoro, 2021).

A osteoporose é causada por perda óssea excessiva, que se deve principalmente à elevação da reabsorção óssea pelos osteoclastos e/ou minimização da formação pelos osteoblastos. Estudos funcionais baseados em especial nos modelos de camundongos ou culturas de células in vitro, têm sido largamente utilizados para determinar a correlação de genes novos ou bem conhecidos com a osteoclastogênese, osteoblastogênese e fenótipos ósseos (Xu, et al., 2010; Ferraz, et al., 2021; Mota, et al., 2021).

A osteoporose pode ser primária (idiopática) ou secundária, sendo a primária dividida em tipo I e II. No tipo I, também conhecida como pós-menopausa, tem-se a perda óssea rápida e ocorre em mulheres que entraram recentemente neste período. Afeta principalmente o osso trabecular e está relacionada a fraturas das vértebras e do rádio distal. Já o tipo II, está relacionada ao envelhecimento e ocorre devido à deficiência crônica de cálcio, aumento da atividade do hormônio da paratireoide e diminuição da formação óssea (Riggs \& Melton, 1983). E a osteoporose secundária é causada por processos inflamatórios, como artrite reumatoide; alterações endócrinas (hipertireoidismo e doença adrenal); mieloma múltiplo; e por uso de drogas como corticoides, uma vez que eles inibem a absorção intestinal do cálcio e elevam a sua excreção na urina, minimizando a formação osteoblástica e elevando a reabsorção osteoclástica (Lane \& Nydick, 1999).

Os osteoclastos de reabsorção óssea possuem gênese hematopoiética e são oriundos de células da linhagem monócitomacrófago (Roodman, 2006). Ramalho e colaboradores (2000) demonstram que há discordância no que diz respeito ao estágio onde a via de diferenciação de monócitos-macrófagos se separam daquela dos osteoclastos. Os autores trouxeram três teorias: 1 - Os osteoclastos são derivados de um progenitor específico, o CFU-O (Unidades formadoras de colônias de osteoclastos), gerado de CFU-S (Unidades formadoras de colônias de haste); 2 - Os osteoclastos são derivados de CFU-GM (Unidades formadoras de Colônia de granulócitos e macrófagos), um precursor comum de osteoclastos, bem como de granulócitos e macrófagos; 3 - Os osteoclastos serão derivados de macrófagos teciduais e monócitos circulantes.

A primeira teoria é improvável, uma vez que nenhum precursor de osteoclastos específico foi comprovado até o momento. A segunda teoria é mais provável porque a capacidade do precursor CFU-GM de se diferenciar em osteoclastos quanto em monócitos circulantes ou macrófagos teciduais foi confirmada. Alguns autores comprovaram a terceira teoria, na qual os osteoclastos podem ser formados não apenas por células imaturas da linhagem monócito-macrófago, mas também por monócitos circulantes e macrófagos teciduais quando o ambiente fornecido é suficiente. Portanto, as duas últimas teorias constituem caminhos possíveis para o recrutamento de osteoclastos (fisiológica e patologicamente) (Ramalho, et al., 2000; Rezende, 2021).

Em ossos periféricos adultos, como os ossos do quadril, a medula local não é uma fonte importante de produção de osteoclastos, na verdade, todos os osteoclastos que atuam nessas áreas vêm principalmente de monócitos que migram para o osso através da circulação periférica (Parfitt, et al., 1996; Parfitt, 2001). A osteoclastogênese está ligada à diferenciação de monócitos em osteoclastos, e em ossos periféricos adultos, como o osso do quadril, a osteoclastogênese ocorre somente após os monócitos do sangue periférico (MSP) migrarem através da superfície endotelial do sangue para o osso (Roodman, 2006).

Depois de migrar do sangue periférico para o osso, os monócitos se diferenciam e se fundem em osteoclastos imaturos multinucleados, que são então ativados para se tornarem osteoclastos maduros no local da reabsorção óssea (Roodman, 2006). É muito difícil coletar um grande número de osteoclastos de humanos para experimentos; no entanto, é relativamente fácil coletar grandes quantidades de MSP, que são precursores dos osteoclastos (Roodman, 2006; Rezende, 2021).

Estudos constataram várias citocinas sintetizadas pelos MSP (como por exemplo, as interleucinas (IL) 1 e 6, bem como o fator de necrose tumoral $\alpha$ ), que são importantes para a diferenciação, ativação e apoptose dos osteoclastos (Cohen- 
Solal, et al., 1993; Pacifici, 1996; Cohen-Solal, et al., 1998). A expressão diferencial de genes em MSP estão relacionadas às variações na baixa densidade mineral óssea (BDMO) (Liu, et al., 2005; Deng, et al., 2008; Farber, 2010; Rezende, 2021).

E de acordo com os resultados de Deng e colaboradores (2011) foi exposto que são necessárias a utilização de MSP como um tipo de célula modelo para pesquisar a função de genes relacionados ao risco de osteoporose humana. Uma vez que, dissecar mudanças nos níveis de expressão de proteínas em precursores de osteoclastos primários humanos, como por exemplo os MSP, em condições normais versus doença é uma tática potencialmente eficaz que pode ser usada para identificar genes funcionalmente relacionados a osteoclastogênese in vivo em humanos.

As anexinas são proteínas reguladas pelo $\mathrm{Ca}^{2+}$, fosfolipídicas e ligadas à membrana. Recebem o nome da palavra grega "anexar", devido a sua habilidade de ligar a sua membrana em estruturas ou em outras membranas (Gerke, Creutz, Moss, 2005). Entre os 12 membros da família anexina (A1-A11 e A13) identificados em vertebrados, todos, exceto um (A6) têm um domínio central altamente homólogo ( 30-35 quilodaltons), que contém quatro repetições de hélice alfa múltiplas com ligação potencial de $\mathrm{Ca}^{2+}$ e um domínio N-terminal ( 3 quilodaltons), específico para cada membro da família (Rescher, Gerke, 2004; Hajjar, 2015).

A anexina é onipresente em toda a árvore filogenética e é evolutivamente antiga. A anexina A2 (ANXA2) é um dos membros da superfamília da anexina mais amplamente estudados (Rescher \& Gerke, 2004). O gene ANXA2 é produzido por uma variedade de tipos de células, incluindo as endoteliais, trofoblásticas, epiteliais e tumorais, bem como células imunes inatas, como macrófagos, monócitos e dendríticas. O ANXA2 é capaz de existir na forma monomérica ou heterotetramérica (Cesarman, Guevara, Hajjar, 1994; Hajjar, Jacovina, Chacko, 1994).

O heterotetrâmero (A2 - S100A10) 2 consiste em duas cópias cada de ANXA2 e da proteína S100A10, também conhecida como p11. O gene ANXA2 está localizado no citoplasma e na superfície celular, e seu papel é amplo e específico neste local. Na superfície das células endoteliais, o complexo (A2 • S100A10) 2 combina componentes do sistema fibrinolítico, plasminogênio e ativador do plasminogênio tecidual (tPA) para acelerar a ativação da serina protease plasmina (Cesarman, Guevara, Hajjar, 1994; Hajjar, Jacovina, Chacko, 1994). Como protease fibrinolítica primária, a plasmina permite a degradação da fibrina e a angiogênese (Hajjar \& Acharya, 2000; Dassah, et al., 2009). Na célula, o ANXA2 parece ter muitas funções, incluindo a organização de microdomínios de membrana especializados, a promoção de brotamento de vesículas e a regulação de outros eventos dinâmicos de membrana, como fusão, endocitose, biogênese endossômica e reparo de membrana (Deng, et al., 2011).

A regulação positiva do $A N X A 2$, em ambos os níveis de expressão de mRNA e proteína, de MSP em indivíduos com BDMO baixa versus alta, apoia-se fortemente a relevância funcional do gene para a regulação da BDMO in vivo em humanos. Para explorar ainda mais a importância do gene $A N X A 2$ para os fenótipos ósseos, foram avaliadas a ligação entre a osteoporose no nível do DNA o ANXA2. Em amostras de caso controle e população, foram identificados SNPs no gene ANXA2 associados à $\mathrm{BDMO}$ do osso branco do quadril. E deixando evidencias de que o ANXA2 é um gene de suscetibilidade à osteoporose em humanos (Deng, et al., 2011).

Ainda Deng e colaboradores (2011), em sua pesquisa, avaliou os SNPs do gene $A N X A 2$, e associou à variação da BDMO em uma população caucasiana, foram identificados um total de 15 SNPs localizados dentro do gene $A N X A 2$ e cobertos pela matriz Affymetrix, destes, três SNPs (rs7163836, rs11633657 e rs11633619) estavam ligados a BDMO do quadril, bem como a BDMO do colo femoral. Tendo o rs11633619, associado à BDMO do quadril e do colo do fêmur.

Deng e colaboradores (2011) simularam a migração transendotelial, ou seja, a barreira endotelial entre o sangue e o osso, uma vez que os MSP devem migrar através dos capilares para os locais de reabsorção na superfície óssea antes que possam se diferenciar e formar osteoclastos de reabsorção óssea. Visto que o ANXA2 é secretado por MSP humano, com isso, é essencial ter o discernimento do papel da proteína ANXA2 exógena na migração transendotelial de monócitos. E na sua 
pesquisa, foi sugerido que, a ANXA2 extracelular existente no sangue humano é capaz de induzir os monócitos a migrarem para a superfície de reabsorção óssea através do endotélio.

Como também, a ANXA2 extracelular, presente no local de reabsorção óssea do corpo humano, é capaz de promover a migração de monócitos da circulação através do endotélio e atraí-los para o osso para se diferenciarem em osteoclastos. Essas duas atividades podem funcionar sinergicamente para acelerar ainda mais o recrutamento de MSP para o osso. Apoiando o importante papel da proteína ANXA2 na regulação da migração transendotelial de monócitos (Falcone, et al., 2001; Brownstein, et al., 2004; Deng, et al., 2011).

Além do papel de ANXA2 na migração transendotelial de monócitos, evidências de estudos in vitro indicam que ANXA2 também é importante para a formação de osteoclastos e reabsorção óssea. Especificamente, em culturas de medula óssea humana, ANXA2 promove a proliferação e diferenciação de precursores de osteoclastos e aumenta a formação de osteoclastos e a reabsorção óssea (Takahashi, et al., 1994; Menaa, et al., 1999; Li, et al., 2005; Lu, et al., 2006). O ANXA2 pode interagir com o antígeno CD44 na quimiotaxia de células semelhantes aos neutrófilos em resposta ao fator complementar Va in vitro e o anti-ANXA2 parece bloquear essa atividade em neutrófilos humanos (Mcvoy \& Kew, 2005).

Ao mesmo tempo, em comparação com outros leucócitos circulantes, o nível de expressão de ANXA2 em neutrófilos humanos recém-isolados parece ser menor, especialmente em monócitos humanos e macrófagos derivados de monócitos, onde seu nível de expressão é muito alto. Na verdade, o anti-ANXA2 IgG impede a migração de monócitos induzida por citocinas através da matriz extracelular (Brownstein, et al., 2004).

E esta atividade de migração também requer a ativação de plasminogênio dependente de tPA como a serina protease plasmina. Além disso, experimentos de cicatrização de feridas in vitro demonstraram que a perda da expressão de ANXA2 em células epiteliais intestinais leva ao aumento da adesão à matriz celular por meio da integrina $\beta$ e à redução da migração celular, mas não está claro se esse mecanismo é aplicável as células inflamatórias. No entanto, a extensão total das ações do ANXA2 no recrutamento de células inflamatórias in vivo ainda não foi bem elucidada (Rankin, et al., 2013).

\section{Conclusão}

Contudo, as alterações morfofuncionais podem estar ligadas a processos danosos e a diminuição de estabilidade da proteína, dificultando assim a sua ação. Além disso, a compreensão das alterações morfofuncionais e de estabilidade do rs1803909 podem auxiliar na busca por marcadores genéticos e moleculares de diagnóstico precoce para a osteoporose em humanos.

Como também, proporciona a compressão dos possíveis processos de alterações estruturais, funcionais e de estabilidade acometendo assim vias fisiológicas. E mais pesquisas funcionais e trabalhos com metodologia robusta e aprofundada são necessárias para elucidar a função do gene ANXA2 e o impacto in vivo do rs1803909 na gênese da osteoporose humana.

\section{Referências}

Adzhubei, I. A., et al. (2010). A method and server for predicting damaging missense mutations. Nat Methods, 7(4): 248-249.

Blakely, R. D. (2005). Overview: a rare opportunity or just one less reason to be depressed. Neuron, 48, 701-2.

Bromberg, Y. \& Rost, B. (2007). SNAP: predict effect of non-synonymous polymorphisms on function. Nucleic Acids Res. 35(11): 3823-3835.

Brownstein, C., et al. (2004). Annexin II mediates plasminogen-dependent matrix invasion by human monocytes: Enhanced expression by macrophages. Blood., 103, 317-324.

Cesarman, G. M., Guevara, C. A., Hajjar, K. A. (1994). An endothelial cell receptor for plasminogen/tissue plasminogen activator (t-PA). II. Annexin IImediated enhancement of t-PA-dependent plasminogen activation. J. Biol. Chem., 269, 21198-21203. 
Cheng, J., Randall, A., Baldi, P. (2006). Prediction of protein stability changes for single-site mutations using support vector machines. Proteins, 62(4): 11251132 .

Choi, Y. \& Chan A. P. (2015). PROVEAN web server: a tool to predict the functional effect of amino acid substitutions and indels. Bioinformatics, 31(16): 2745-2747.

Chorley, B. N., et al. (2008). Discovery and verification of functional single nucleotide polymorphisms in regulatory genomic regions: current and developing technologies. Mutat Res, 659, 147-57.

Cohen-Solal, M. E., et al. (1993). Peripheral monocyte culture supernatants of menopausal women can induce bone resorption: involvement of cytokines. $J$. Clin. Endocrinol. Metab., 77, 1648-1653.

Cohen-Solal, M. E., et al. (1998). Increased bone resorbing activity of peripheral monocyte culture supernatants in elderly women. J. Clin. Endocrinol. Metab., $83,1687-1690$.

Coughlan, T., \& Dockery, F. (2014). Osteoporosis and fracture risk in older people. Clinical medicine, 14, 187-191.

Dassah, M., et al. (2009). The Endothelial Cell Annexin A2 System and Vascular Fibrinolysis. Gen. Physiol. Biophys., $28,20-28$.

Jong, M. C., et al. (2010). CD44 Expression Predicts Local Recurrence After Radiotherapy In Larynx Cancer. Clin. Cancer Res., 16, $5329-5338$.

Deng, F. Y., et al. (2008). Proteomic analysis of circulating monocytes in Chinese premenopausal females with extremely discordant bone mineral density. Proteomics., 8, 4259-4272.

Deng, F. Y., et al. (2011). Peripheral blood monocyte-expressed ANXA2 gene is involved in pathogenesis of osteoporosis in humans. Molecular \& cellular proteomics: $M C P, 10$, M111.011700.

Drysdale, C. M., et al. (2000). Complex promoter and coding region beta 2-adrenergic receptor haplotypes alter receptor expression and predict in vivo responsiveness. Proc Natl Acad Sci., USA, 97, 10483-8.

Falcone, D. J., et al. (2001). Plasminogen-mediated matrix invasion and degradation by macrophages is dependent on surface expression of annexin II. Blood., 97, 777-784.

Farber, C. R. (2010). Identification of a gene module associated with BMD through the integration of network analysis and genome-wide association data. $J$. Bone Miner. Res., 25, 2359-2367.

Ferraz, C. P.; Nolasco, R. W. M.; Amaral, P. A. S. \& Pereira, L. C. (2021). Osteoporosis and oral health: literature review. Research, Society and Development, 10(15), e275101522930. https://doi.org/10.33448/rsd-v10i15.22930

Frazer, K. A., et al. (2007). International HapMap Consortium. A second generation human haplotype map of over 3.1 million SNPs. Nature, 449 , 851-61.

Gerke, V., Creutz, C. E., Moss, S. E. (2005). Annexins: Linking Ca2+ signalling to membrane dynamics. Nat. Rev. Mol. Cell Biol., 6, 449-461.

Hajjar, K. A. \& Acharya, S. S. (2000). Annexin II and Regulation of Cell Surface Fibrinolysis. Ann. N. Y. Acad. Sci., 902, 265-271.

Hajjar, K. A. (2015). The Biology of Annexin A2: From Vascular Fibrinolysis to Innate Immunity. Trans. Am. Clin. Climatol. Assoc., 126, 144-155.

Hajjar, K. A.; Jacovina, A. T.; Chacko, J. (1994). An endothelial cell receptor for plasminogen/tissue plasminogen activator. I. Identity with annexin II. J. Biol. Chem., 269, 21191-21197.

Hedhli, N. D. J., et al. (2012). The annexin A2/S100A10 system in health and disease: emerging paradigms. J.Biomed.Biotechnol. p. $4062-73$.

Karg, K., et al. (2011). The serotonin transporter promoter variant (5-HTTLPR), stress, and depression metaanalysis revisited: evidence of genetic moderation. Arch Gen Psychiatry, 68, 444-54.

Kesisis, G., et al. (2010). Biological markers in breast cancer prognosis and treatment. J. Buon., 15, 447-454.

Kuo, Y. B., et al. (2011). Identification of Phospholipid Scramblase 1 as a biomarker and it's prognostic value for Colorectal Cancer. Mol. Med., $17,41-47$.

Lane, J. M. \& Nydick, M. (1999). Osteoporosis: Current modes of prevention and treatment. J Am Acad Ortho Surg, 7, $19-31$.

Li, F., et al. (2005). Annexin II stimulates RANKL expression through MAPK. J. Bone Miner. Res., 20, 1161-1167.

Liao, P-Y. \& Lee, K. H. (2010). From SNPs to functional polymorphism: the in sight into biotechnology applications. Biochem Eng J, 49 , 149-58.

Liu, Y. Z., et al. (2005). A novel pathophysiological mechanism for osteoporosis suggested by an in vivo gene expression study of circulating monocytes. $J$. Biol. Chem., 280, 29011-29016.

Lu, G., et al. (2006). Cloning and characterization of the annexin II receptor on human marrow stromal cells. J. Biol. Chem., 281, 30542-30550.

Mathe, E., et al. (2006). Abordagens computacionais para prever o efeito biológico de mutações missense de p53: uma comparação de três métodos baseados em análise de sequência. Nucleic Acids Res. 34 (5): 1317-25.

Mcvoy, L. A. \& Kew, R. R. (2005). CD44 and Annexin A2 Mediate the C5a Chemotactic Cofactor Function of the Vitamin D Binding Protein. J. Immunol., $175,4754-4760$. 
Research, Society and Development, v. 11, n. 1, e8511124356, 2022

(CC BY 4.0) | ISSN 2525-3409 | DOI: http://dx.doi.org/10.33448/rsd-v11i1.24356

Menaa, C., et al. (1999). Annexin II increases osteoclast formation by stimulating the proliferation of osteoclast precursors in human marrow cultures. J. Clin. Invest., 103, 1605-1613.

Mota, P. H. R., et al. (2021). The influence of osteoporosis on implant dentistry. Research, Society and Development, 10(15), e82101522976. https://doi.org/10.33448/rsd-v10i15.22976

Ng, P. C. \& Henikoff S. (2003). SIFT: Predicting amino acid changes that affect protein function. Nucleic Acids Res. 31(13): $3812-3814$.

Pacifici, R. (1996). Estrogen, cytokines, and pathogenesis of postmenopausal osteoporosis. J. Bone Miner. Res., 11, 1043-1051.

Parfitt, A. M. (2001). Skeletal heterogeneity and the purposes of bone remodelling: implications for the understanding of osteoporosis. In: Marcus R. Zfeldman D. Kelsey J. Osteoporosis. Academic Press, San Diego, 433-444.

Parfitt, A. M., et al. (1996). A new model for the regulation of bone resorption, with particular reference to the effects of bisphosphonates. J. Bone Miner. Res., $11,150-159$.

Ramalho, A. C. R., et al. (2000). Por Que Estrógeno e Raloxifeno Melhoram a Densidade Mineral Óssea? Mecanismo de Ação do Estrógeno e de Um Modulador Seletivo do Receptor de Estrógeno (SERM) no Osso. Arq. Bras. Endocrinol. Metab. 44(6), 471-482.

Rankin, C. R., et al. (2013). Annexin A2 Regulates $\beta 1$ Integrin Internalization and Intestinal Epithelial Cell Migration. J. Biol. Chem., 288, 15229-15239.

Rescher, U. \& Gerke, V. (2004). Annexins unique membrane binding proteins with diverse functions. J. Cell Sci., 117, 2631-2639.

Rezende, R. B. \& Teodoro, L. (2021). Impacto Do Polimorfismo RS121913578 do Gene Mrt Associado ao Câncer de Tireoide: Impact of the RS121913578 Polymorphism of the Mrt Gene Associated with Thyroid Cancer. Archives of Health, 2(4), 1101-1104.

Rezende, R. B. (2021). Hemodynamic Dysfunctions in COVID-19 Patients. Research, Society and Development, 10(12), e111101220323. https://doi.org/10.33448/rsd-v10i12.20323

Riggs, B. L. \& Melton, L. J. (1983). Evidence for two distinct syndromes of involutional osteoporosis. Am J Med, 75, 899-901.

Roodman, G. D. (2006). Regulation of osteoclast differentiation. Ann. N.Y. Acad. Sci., 1068, 100-109.

Sadee, W., et al. (2011). Pharmacogenomics of the RNA world: structural RNA polymorphisms in drug therapy. Clin Pharmacol Ther, 89, 355-65.

Takahashi, S., et al. (1994). Cloning and identification of annexin II as an autocrine/paracrine factor that increases osteoclast formation and bone resorption. $J$. Biol. Chem., 269, 28696-28701.

Tavtigian, S. V., et al. (2006). An Analysis of Unclassified Missense Substitutions in Human BRCA1. Cancro Familiar. 5 (1): $77-88$.

Xu, X. H., et al. (2010). Molecular genetic studies of gene identification for osteoporosis: the 2009 update. Endocr. Rev., 31, 447-505.

Zhang, X., et al. (2005). Loss-of-function mutation in tryptophan hydroxylase-2 identified in unipolar major depression. Neuron, 45, 11-16. 